\title{
DOCUMENTAÇÃO DOS CUIDADOS DE ENFERMAGEM DURANTE O PROCESSO DE MORRER - COMO SE DIFERENCIAM OS ENFERMEIROS DE REABILITAÇÃO?
}

\author{
NURSING CARE DOCUMENTATION DURING THE DYING PROCESS - HOW DO THE REHABILITATION NURSES DIFFER?
}

\section{DOCUMENTACIÓN DE LOS CUIDADOS DE ENFERMERÍA DURANTE EL PROCESO DE MUERTE - CÓMO SE DIFERENCIAN LOS ENFERMEROS DE REHABILITACIÓN?}

DOI 10.33194/rper.2019.v2.n2.02.4569 | Submetido 17.03.2019 | Aprovado 02.12.2019

\author{
Maria Filomena Cardoso ${ }^{1,2,3}{ }^{-}$; Maria Manuela Martins ${ }^{4,5}$; Olga Ribeiro ${ }^{4,5}$ \\ 1 - Instituto de Ciências Biomédicas Abel Salazar; 2 - Centro Hospitalar Universitário de São João; 3 - Universidade Fernando \\ Pessoa; 4 - Escola Superior de Enfermagem do Porto; 5 - CINTESIS
}

\section{RESUMO}

Objetivo: analisar os focos/diagnósticos de enfermagem documentados pelos enfermeiros especialistas em enfermagem de reabilitação, durante o processo de morrer em contexto hospitalar.

Método: estudo descritivo, retrospetivo e quantitativo realizado numa instituição hospitalar do norte de Portugal em fevereiro de 2017. Os dados sobre a documentação dos focos/diagnósticos de enfermagem identificados nos clientes que morreram no ano de 2016 foram recolhidos por meio de dois sistemas de informação: SClínico e BICUcare.

Resultados: nos 4115 registos efetuados por 148 enfermeiros especialistas em enfermagem de reabilitação, constatamos que apesar da evolução ocorrida durante a última década, na documentação dos cuidados de enfermagem, sobressaem algumas inquietações sobre qual a prática de cuidados que a mesma evidencia. Decorrente da ênfase colocada na documentação das alterações no domínio da função, os enfermeiros especialistas em enfermagem de reabilitação tendem a subestimar o registo das alterações e das necessidades que emergem das transições vivenciadas pelas pessoas, especificamente durante a morte e os processos de morrer.

Conclusão: Atendendo a que a informação registada contribui para a visibilidade dos cuidados prestados, emerge a necessidade de se adotarem estratégias que resolvam o problema da subdocumentação, nomeadamente perante a morte e os processos de morrer.

Descritores: Morte; Enfermagem; Enfermagem em Reabilitação; Registros Eletrónicos de Saúde; Diagnóstico de Enfermagem

\section{RESUMEN}

Objetivo: analizar los focos / diagnósticos de enfermería documentados por enfermeras especializadas en enfermería de rehabilitación durante el proceso de muerte en un contexto hospitalario.

Método: estudio descriptivo, retrospectivo y cuantitativo realizado en un hospital del norte de Portugal en febrero de 2017. Los datos sobre la documentación de brotes de enfermería / diagnósticos identificados en clientes que fallecieron en 2016 se obtuvieron mediante dos sistemas de información. : SClínico y BICUcare.

Resultados: De los 4115 registros realizados por 148 enfermeras especializadas en enfermería de rehabilitación, descubrimos que a pesar de la evolución que se ha producido durante la última década en la documentación de la atención de enfermería, existen algunas preocupaciones sobre lo que muestra la práctica de atención. Debido al énfasis puesto en documentar los cambios en el campo de la función, las enfermeras especializadas en enfermería de rehabilitación tienden a subestimar el registro de cambios y necesidades que surgen de las transiciones experimentadas por las personas, específicamente durante los procesos de muerte y muerte.

Conclusión: Dado que la información registrada contribuye a la visibilidad de la atención brindada, es necesario adoptar estrategias que resuelvan el problema de la subdocumentación, particularmente frente a los procesos de muerte y muerte.

Palabras Ilave: Muerte; Enfermería; Enfermería de rehabilitación; Registros de salud electrónicos; Diagnóstico de enfermería 


\section{ABSTRACT}

Objective: To analyze the nursing foci / diagnoses documented by nurse specialists in rehabilitation nursing during the process of dying in a hospital context.

Method: descriptive, retrospective and quantitative study conducted at a hospital in northern Portugal in February 2017. Data on documentation of nursing outbreaks / diagnoses identified in clients who died in 2016 were collected using two information systems: SClinic and BICUcare.

Results: From the 4115 records made by 148 nurses who specialize in rehabilitation nursing, we found that despite the evolution that has taken place over the last decade in the documentation of nursing care, there are some concerns about the practice of care that evidences. Due to the emphasis placed on documenting changes in the field of function, specialist nurses in rehabilitation nursing tend to underestimate the record of changes and needs that emerge from the transitions experienced by people, specifically during death and dying processes.

Conclusion: Given that the information recorded contributes to the visibility of the care provided, there is a need to adopt strategies that solve the problem of underdocumentation, particularly in the face of death and dying processes.

Keywords: Death; Nursing; Rehabilitation Nursing; Electronic Health Records; Nursing Diagnosis

\section{INTRODUÇÃO}

Cada ser humano carrega consigo uma representação individual da morte. Esta é elaborada por influência do convívio social, dos meios de comunicação e por particularidades de cada individuo. "É neste cenário de diversidade com relação à morte que se encontram os profissionais de enfermagem, vivendo em constante desafio, uma vez que diariamente permanecem em conflito, lutando pela vida e contra a morte, tomando para si a responsabilidade de salvar, curar ou aliviar, procurando sempre preservar a vida, já que a morte, na maioria das vezes, é vista por estes profissionais como um fracasso, sendo, desta forma duramente combatida"(1:42).

Criamos cada vez mais subespecialidades que vão tentando prolongar mais ou menos artificialmente a beleza, as funções vitais e adiando esse momento que, apesar de todo o empenho e esforço, continua inultrapassável. "Por conseguinte, esse prolongamento implica a necessidade de um cuidar diferenciado, pautado na valorização da qualidade de vida e do bemestar biopsicossocial e espiritual do paciente, visando direcionar a assistência de saúde e, em especial, a de enfermagem, por meio de uma visão holística $e$ integral, revelando-se, assim, um "cuidar humanizado"(2:318).

Os profissionais de saúde em geral, e os enfermeiros de modo particular, são capacitados para ajudar os outros a resolver os seus problemas, para promover a saúde, e incentivar hábitos de vida saudáveis. De facto, não são preparados para aceitar que há limites, que nem sempre vão poder tratar e devolver a saúde à pessoa doente, pelo que têm que investir no bem-estar. E se o referido é notório nos enfermeiros de cuidados gerais, questionamo-nos se também o será no caso dos enfermeiros especialistas.

De acordo com o Regulamento das Competências Comuns do Enfermeiro Especialista(3) ${ }^{(3)}$ são quatro os domínios de competências: responsabilidade profissional, ética e legal; melhoria contínua da qualidade; gestão dos cuidados e desenvolvimento das aprendizagens profissionais.
No âmbito da problemática em estudo, no domínio da responsabilidade profissional, ética e legal, destacamos que o enfermeiro especialista deve promover práticas de cuidados que respeitem os direitos humanos e as responsabilidades profissionais, nomeadamente perante situações potencialmente comprometedoras para os clientes. Como critério de avaliação destacamos o respeito pelos valores, costumes, crenças espirituais, bem como pelas práticas específicas dos indivíduos e grupos em todas as fases do ciclo de vida ${ }^{(3)}$.

De acordo com o mesmo regulamento, no domínio da melhoria da qualidade, considerando a gestão do ambiente centrado na pessoa como condição imprescindível para a efetividade terapêutica e para a prevenção de incidentes, o enfermeiro atua proativamente promovendo a envolvência adequada ao bem-estar e gerindo o risco. Em relação aos critérios de avaliação, destacamos a sensibilidade, a consciência e o respeito em relação às necessidades espirituais do indivíduo/grupo e o envolvimento da família e outros no sentido de assegurar que as necessidades culturais $\mathrm{e}$ espirituais sejam satisfeitas ${ }^{(3)}$.

Já no âmbito das competências específicas do Enfermeiro Especialista em Enfermagem de

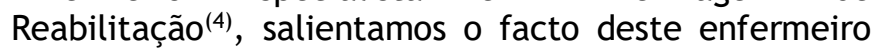
especialista cuidar de pessoas com necessidades especiais, ao longo de todo o ciclo de vida, em todos os contextos da prática de cuidados.

Assim, partindo do pressuposto que o exercício profissional dos enfermeiros especialistas em enfermagem de reabilitação deve estar sustentado nos instrumentos reguladores do exercício profissional, bem como nos referenciais teóricos de enfermagem ${ }^{(5)}$, questionamo-nos sobre a visibilidade desta área de especialidade, no âmbito dos registos efetuados nos sistemas de informação, nomeadamente na assistência às pessoas em processo de morrer.

Importa referir que previamente ao aparecimento dos sistemas de informação informatizados, os registos de enfermagem, como tradicionalmente são nomeados pelos enfermeiros, caracterizavam-se pela utilização de texto livre com o objetivo de produzir narrativas dos cuidados prestados, cujo interesse se circunscrevia à produção de documentos com valor legal ${ }^{(6)}$. Nos dias de 
hoje, decorrente da evolução verificada, apesar de ser notória a qualidade dos registos, será que os mesmos refletem em todas as situações os cuidados prestados no âmbito da especialização em enfermagem de reabilitação?

Partindo desta inquietação, integrado no projeto de pesquisa mais alargado "Viver a Morte: desafio da profissão de enfermagem", colocamos a seguinte questão: será que a documentação efetuada pelos enfermeiros com especialização em enfermagem de reabilitação expressa a sua prática, durante a morte e o processo de morrer?

Em consonância com o referido, foi definido como objetivo deste estudo analisar os focos/diagnósticos de enfermagem documentados pelos enfermeiros especialistas em enfermagem de reabilitação, no cuidar das pessoas perante a morte e os processos de morrer.

\section{MÉTODO}

Estudo descritivo, retrospetivo e quantitativo, realizado nos serviços dos departamentos de medicina, cirurgia e medicina intensiva, de um hospital universitário do norte de Portugal.

Os dados sobre a documentação dos focos/diagnósticos de enfermagem identificados nos clientes que morreram no ano de 2016 foram recolhidos, em fevereiro de 2017, por meio de dois sistemas de informação: SClínico e BICUcare estruturados com base na Classificação Internacional para a Prática de Enfermagem $\left(\right.$ CIPE $\left.^{\circledR}\right)$ versão $\beta 2$. Foram analisados 36281 registos efetuados por 1270 enfermeiros. Posteriormente, na sequência de uma amostragem não probabilística e intencional, foram analisados 9202 registos realizados por 320 enfermeiros especialistas, dos quais 4115 foram efetuados por 148 enfermeiros especialistas em enfermagem de reabilitação. Foram definidos como critérios de exclusão: registos sobre clientes com idade inferior a 18 anos, registos sem identificação clara da autoria do enfermeiro e registos realizados fora das áreas de medicina, cirurgia e medicina intensiva.

Para o tratamento dos dados usámos o programa estatístico Statistical Package for the Social Sciences (SPSS), versão 22.0, sendo que de acordo com a natureza das variáveis e os objetivos do estudo, recorremos à estatística descritiva.

\section{RESULTADO}

Dos 320 enfermeiros que possuem uma especialização em enfermagem e que efetuaram registos nos doentes que faleceram, para além do predomínio do género feminino $(65,5 \%)$, a especialização em enfermagem de reabilitação foi a que surgiu com maior frequência: 148 enfermeiros $(46,3 \%)$.

A idade dos enfermeiros com a especialização em enfermagem de reabilitação varia entre os 25 e os 55 anos, sendo a média 36,8 anos.
Relativamente aos serviços onde os enfermeiros especialistas em enfermagem de reabilitação exercem funções, predominam os serviços dos departamentos de cirurgia e medicina (Tabela 1). A especialização em enfermagem médico-cirúrgica foi mais frequente nos enfermeiros que exerciam a sua atividade profissional no departamento de medicina intensiva.

\begin{tabular}{|r|c|c|c|c|c|c|c|c|}
$\begin{array}{r}\text { Departa- } \\
\text { mentos }\end{array}$ & \multicolumn{2}{|c|}{ Cirurgia } & \multicolumn{2}{|c|}{ Medicina } & $\begin{array}{r}\text { Medicina } \\
\text { Intensiva }\end{array}$ & \multicolumn{2}{|c|}{ Total } \\
\cline { 2 - 8 } $\begin{array}{r}\text { Área de } \\
\text { Especializaçãa }\end{array}$ & $\mathrm{n}$ & $\%$ & $\mathrm{n}$ & $\%$ & $\mathrm{n}$ & $\%$ & $\mathrm{n}$ & $\%$ \\
\hline $\begin{array}{c}\text { Enfermeiros } \\
\text { especialistas em } \\
\text { enfermagem de } \\
\text { reabilitação }\end{array}$ & 37 & 56,1 & 64 & 52,5 & 47 & 35,6 & 148 & 46,3 \\
\hline
\end{tabular}

Tabela 1 - Distribuição dos Enfermeiros com Especialização em Enfermagem de Reabilitação nos Departamentos

A distribuição dos registos relativos ao foco Indivíduo efetuados pelos enfermeiros com especialização em enfermagem de reabilitação, encontra-se explanada na Tabela 2.

\begin{tabular}{|c|c|c|c|c|c|c|c|c|}
\hline \multirow{2}{*}{$\begin{array}{l}\text { Departa- } \\
\text { goco re- } \\
\text { gistado } \mathrm{p} / \mathrm{ER}\end{array}$} & \multicolumn{2}{|c|}{ Cirurgia } & \multicolumn{2}{|c|}{ Medicina } & \multicolumn{2}{|c|}{$\begin{array}{l}\text { Medicina } \\
\text { Intensiva }\end{array}$} & \multicolumn{2}{|c|}{ Total } \\
\hline & $\mathrm{n}$ & $\%$ & $n$ & $\%$ & $\mathrm{n}$ & $\%$ & $n$ & $\%$ \\
\hline Indivíduo & 560 & 55,1 & 2165 & 53,4 & 1390 & 33,6 & 4115 & 44,7 \\
\hline
\end{tabular}

Tabela 2 - Registos dos Enfermeiros com Especialização em Enfermagem de Reabilitação no âmbito do Foco Indivíduo

Relativamente ao foco Função foram efetuados pelos enfermeiros com especialização em enfermagem de reabilitação, 2408 (43,0\%) registos, distribuídos pelas três áreas, conforme o enunciado na Tabela 3.

\begin{tabular}{|c|c|c|c|c|c|c|c|c|}
\hline \multirow{2}{*}{$\begin{array}{l}\text { Departa } \\
\text { mento } \\
\text { goco re- } \\
\text { gistado } \mathrm{p} / \mathrm{ER}\end{array}$} & \multicolumn{2}{|c|}{ Cirurgia } & \multicolumn{2}{|c|}{ Medicina } & \multicolumn{2}{|c|}{$\begin{array}{l}\text { Medicina } \\
\text { Intensiva }\end{array}$} & \multicolumn{2}{|c|}{ Total } \\
\hline & $\mathrm{n}$ & $\%$ & $n$ & $\%$ & $n$ & $\%$ & $\mathrm{n}$ & $\%$ \\
\hline Função & 286 & 53,8 & 1126 & 57,0 & 996 & 32,2 & 2408 & 43,0 \\
\hline
\end{tabular}

Tabela 3 - Registos dos Enfermeiros com Especialização em Enfermagem de Reabilitação no âmbito do Foco Função

A distribuição dos registos relativos às áreas de atenção adstritas ao foco Função pode ser analisada no Quadro 1. Realçamos que existem quatro focos (Desenvolvimento Físico, Metabolismo, Nutrição e Reparação), sem nenhum registo.

0 número reduzido de registos dos enfermeiros com diferentes especializações, nos serviços dos departamentos de medicina e cirurgia, impede a realização de qualquer teste estatístico pois, com efeito, nestas duas áreas quase só existem registos dos enfermeiros especialistas em enfermagem de reabilitação. É apenas possível observar que a distribuição dos registos eletrónicos dos enfermeiros detentores das diferentes especializações, nos departamentos de medicina e cirurgia é muito semelhante, distinguindo-se claramente da distribuição do departamento de medicina intensiva. Com efeito, neste último departamento, a especialização em 
Enfermagem Médico-Cirúrgica tem maior representatividade, sucedendo o inverso com a Enfermagem de Reabilitação. Importa referir que neste estudo as restantes especializações quase não têm expressão.

\begin{tabular}{|c|c|c|c|c|c|c|c|c|}
\hline \multirow[b]{2}{*}{ Função } & \multicolumn{2}{|c|}{ Cirurgia } & \multicolumn{2}{|c|}{ Medicina } & \multicolumn{2}{|c|}{$\begin{array}{l}\text { Medicina } \\
\text { Intensiva }\end{array}$} & \multicolumn{2}{|c|}{ Total } \\
\hline & $n$ & $\%$ & $\mathrm{n}$ & $\%$ & $\mathrm{n}$ & $\%$ & $\mathrm{n}$ & $\%$ \\
\hline $\begin{array}{l}\text { Atividade } \\
\text { Motora } \\
\end{array}$ & 63 & 92,6 & 276 & 95,8 & 149 & 46,1 & 488 & 71,9 \\
\hline Circulação & 7 & 53,8 & 12 & 36,4 & 49 & 28,0 & 68 & 30,8 \\
\hline $\begin{array}{l}\text { Desenvolvi- } \\
\text { mento Físico }\end{array}$ & 0 & --- & 0 & --- & 0 & --- & 0 & --- \\
\hline Digestão & 6 & 66,7 & 41 & 77,4 & 28 & 33,3 & 75 & 51,4 \\
\hline Eliminação & 8 & 72,7 & 13 & 41,9 & 82 & 28,2 & 103 & 30,9 \\
\hline Metabolismo & 0 & --- & 0 & --- & 0 & --- & 0 & --- \\
\hline Nutrição & 0 & $\cdots$ & 0 & $\cdots$ & 0 & $-\cdots$ & 0 & $-\cdots$ \\
\hline Reparação & 0 & --- & 0 & --- & 0 & --- & 0 & --- \\
\hline Respiração & 64 & 84,2 & 259 & 77,3 & 145 & 36,3 & 468 & 57,4 \\
\hline Sensações & 15 & 32,6 & 16 & 28,1 & 79 & 26,5 & 110 & 27,4 \\
\hline $\begin{array}{l}\text { Sistema } \\
\text { Imunitário }\end{array}$ & 1 & 100,0 & 1 & 20,0 & 0 & 0,0 & 2 & 20,0 \\
\hline Tegumento & 111 & 40,8 & 488 & 44,4 & 446 & 30,7 & 1045 & 37,0 \\
\hline $\begin{array}{l}\text { Temperatura } \\
\text { Corporal }\end{array}$ & 6 & 46,2 & 6 & 28,6 & 6 & 31,6 & 18 & 34,0 \\
\hline $\begin{array}{l}\text { Volume de } \\
\text { Líquidos }\end{array}$ & 5 & 26,3 & 14 & 29,2 & 12 & 25,0 & 31 & 27,0 \\
\hline
\end{tabular}

Quadro 1 - Registos dos Enfermeiros com Especialização em Enfermagem de Reabilitação no âmbito dos focos adstritos à Função

Os registos relativos ao foco Tegumento efetuados pelos enfermeiros com especialização em enfermagem de reabilitação são os mais frequentes nos serviços dos departamentos de medicina (488 registos - 44,4\%), medicina intensiva (446 registos - 30,7\%) e cirurgia (111 registos - 40,8\%).

Os registos em relação ao foco Atividade Motora, efetuados pelos enfermeiros com especialização em enfermagem de reabilitação são também um dos maioritários, constatando-se a existência de 488 registos $(71,9 \%)$. O maior número destes registos verifica-se nos serviços do departamento de medicina (276 registos - 95,8\%).

Os registos efetuados pelos enfermeiros com especialização em enfermagem de reabilitação são ainda em número elevado no caso dos focos Respiração (468 registos - 57,4\%), Sensação (110 registos - 57,4\%), Eliminação (103 registos - 30,9\%) e Digestão (75 registos - 51,4\%). No que se refere o foco Respiração, os enfermeiros com especialização em enfermagem de reabilitação e que exercem a sua atividade em serviços do departamento de medicina são os que mais registam no âmbito desta área de atenção (259 registos - 77,3\%).
No foco Circulação os registos efetuados pelos enfermeiros com especialização em enfermagem de reabilitação foram 68 (30,8\%). Destes 68 registos, 49 foram efetuados no departamento de medicina intensiva.

A distribuição dos registos relativos ao foco Volume de líquidos efetuados pelos enfermeiros com especialização em enfermagem de reabilitação corresponde apenas a 31 registos $(27,0 \%)$. Relativamente ao foco Temperatura corporal, os enfermeiros com especialização em enfermagem de reabilitação efetuaram 18 registos (34,0\%). No que concerne à distribuição dos registos efetuados pelos enfermeiros com especialização em enfermagem reabilitação em relação ao foco Sistema Imunitário, constatamos que só existe um registo na área Cirúrgica e outro na área Médica.

Quanto aos registos relativos à Pessoa (Tabela 4), segundo as áreas de especialização, os enfermeiros especialistas em enfermagem de reabilitação, são os que apresentam um maior número de registos (1707 registos - 47,4\%), nomeadamente nos serviços do departamento de medicina.

\begin{tabular}{|r|c|c|c|c|c|c|c|c|}
\hline $\begin{array}{r}\text { Departa- } \\
\text { mentos }\end{array}$ & \multicolumn{2}{c|}{ Cirurgia } & \multicolumn{2}{|c|}{ Medicina } & \multicolumn{2}{r|}{$\begin{array}{r}\text { Medicina } \\
\text { Intensiva }\end{array}$} & \multicolumn{2}{|c|}{ Total } \\
\cline { 2 - 9 } $\begin{array}{r}\text { Foco re- } \\
\text { gistado p/ER }\end{array}$ & $\mathrm{n}$ & $\%$ & $\mathrm{n}$ & $\%$ & $\mathrm{n}$ & $\%$ & $\mathrm{n}$ & $\%$ \\
\hline Pessoa & 274 & 56,6 & 1039 & 50,0 & 394 & 38,0 & 1707 & 47,4 \\
\hline
\end{tabular}

Tabela 4 - Registos dos Enfermeiros com Especialização em Enfermagem de Reabilitação no âmbito do Foco Pessoa

Os registos relativos ao foco Ação (Tabela 5) efetuados pelos enfermeiros com especialização em enfermagem de reabilitação foram 1618 (46,7\%).

\begin{tabular}{|c|c|c|c|c|c|c|c|c|}
\hline \multirow{2}{*}{$\begin{array}{l}\text { Departa- } \\
\text { mentos } \\
\text { goco re- }\end{array}$} & \multicolumn{2}{|c|}{ Cirurgia } & \multicolumn{2}{|c|}{ Medicina } & \multicolumn{2}{|c|}{$\begin{array}{l}\text { Medicina } \\
\text { Intensiva }\end{array}$} & \multicolumn{2}{|c|}{ Total } \\
\hline & $\mathrm{n}$ & $\%$ & $n$ & $\%$ & $n$ & $\%$ & $n$ & $\%$ \\
\hline Ação & 258 & 56,2 & 987 & 49,4 & 373 & 37,1 & 1618 & 46,7 \\
\hline
\end{tabular}

Tabela 5 - Registos dos Enfermeiros com Especialização em Enfermagem de Reabilitação no âmbito do Foco Ação

$\mathrm{Na}$ distribuição dos registos relativos ao foco Ação Interdependente, os registos dos enfermeiros com especialização em enfermagem de reabilitação (Tabela 6) são maioritários (48 registos - 75,0\%)

\begin{tabular}{|r|c|c|c|c|c|c|c|c|}
\hline $\begin{array}{r}\text { Departa- } \\
\text { mentos }\end{array}$ & \multicolumn{2}{|c|}{ Cirurgia } & \multicolumn{2}{|c|}{ Medicina } & $\begin{array}{r}\text { Medicina } \\
\text { Intensiva }\end{array}$ & \multicolumn{2}{|c|}{ Total } \\
\cline { 2 - 9 } $\begin{array}{l}\text { Foco re- } \\
\text { gistado p/ ER }\end{array}$ & $\mathrm{n}$ & $\%$ & $\mathrm{n}$ & $\%$ & $\mathrm{n}$ & $\%$ & $\mathrm{n}$ & $\%$ \\
\hline $\begin{array}{c}\text { Ação } \\
\text { Interdependente }\end{array}$ & 3 & 60,0 & 44 & 78,6 & 1 & 33,3 & 48 & 75,0 \\
\hline
\end{tabular}

Tabela 6 - Registos dos Enfermeiros com Especialização em Enfermagem de Reabilitação no âmbito do Foco Ação Interdependente 
Os registos dos diferentes focos correspondentes à área Ação Interdependente podem ser analisados no Quadro 2.

Importa referir que os registos relativos ao foco Comunicação (8 registos - 72,7\%), Interação de Papéis (9 registos - 52,9\%) e Interação Social (31 registos - $86,1 \%$ ) são maioritariamente efetuados pelos enfermeiros com especialização em enfermagem de reabilitação. Não se identificaram registos relativos ao foco Interação Sexual, nem ao foco Prestar Cuidados.

\begin{tabular}{|c|c|c|c|c|c|c|c|c|}
\hline \multirow{2}{*}{$\begin{array}{l}\text { Departa- } \\
\text { goco re- } \\
\text { gistado } \mathrm{p} / \mathrm{ER}\end{array}$} & \multicolumn{2}{|c|}{ Cirurgia } & \multicolumn{2}{|c|}{ Medicina } & \multicolumn{2}{|c|}{$\begin{array}{l}\text { Medicina } \\
\text { Intensiva }\end{array}$} & \multicolumn{2}{|c|}{ Total } \\
\hline & $\mathrm{n}$ & $\%$ & $n$ & $\%$ & $\mathrm{n}$ & $\%$ & $\mathrm{n}$ & $\%$ \\
\hline Comunicação & 0 & --- & 7 & 77,8 & 1 & 50,0 & 8 & 72 \\
\hline $\begin{array}{c}\text { Interação de } \\
\text { Papeis }\end{array}$ & 3 & 100,0 & 6 & 46,2 & 0 & 0,0 & 9 & 52 \\
\hline Interação Sexual & 0 & & 0 & & 0 & $-\cdots$ & 0 & \\
\hline Interação Social & 0 & -- & 31 & 91,2 & 0 & - & 31 & \\
\hline Prestar Cuidados & 0 & $\mid-\ldots$ & 0 & -.... & 0 & $-\cdots$ & 0 & \\
\hline
\end{tabular}

Quadro 2 - Registos dos Enfermeiros com Especialização em Enfermagem de Reabilitação no âmbito dos focos adstritos à Ação Interdependente

Em relação aos registos efetuados pelos enfermeiros relativamente ao foco Ação Realizada pelo Próprio, segundo as áreas de especialização, os enfermeiros especialistas em enfermagem de reabilitação são os que apresentam um maior número de registos (1570 registos - 46,2\%). No quadro 3 podem ser analisadas as áreas de atenção em relação ao foco Ação Realizada pelo Próprio.

No que concerne aos registos efetuados no âmbito do Autocuidado, segundo as áreas de especialização, os enfermeiros especialistas em enfermagem de reabilitação são os que apresentam um número mais elevado de registos (1570 registos - 46,2\%).

No que refere ao foco Estilos de Vida, nenhum dos enfermeiros com especialização em enfermagem efetuou registos, existindo apenas 6 registos efetuados por enfermeiros de cuidados gerais.

\begin{tabular}{|c|c|c|c|c|c|c|c|c|}
\hline \multirow{2}{*}{$\begin{array}{l}\text { Departa- } \\
\text { mentos } \\
\text { gistado } \mathrm{p} / \mathrm{ER}\end{array}$} & \multicolumn{2}{|c|}{ Cirurgia } & \multicolumn{2}{|c|}{ Medicina } & \multicolumn{2}{|c|}{$\begin{array}{l}\text { Medicina } \\
\text { Intensiva }\end{array}$} & \multicolumn{2}{|c|}{ Total } \\
\hline & $\mathrm{n}$ & $\%$ & $\mathrm{n}$ & $\%$ & $n$ & $\%$ & $\mathrm{n}$ & $\%$ \\
\hline Auto-cuidado & 255 & 56,2 & 943 & 48,5 & 372 & 37,1 & 1570 & 46,2 \\
\hline $\begin{array}{c}\text { Cuidado } \\
\text { Doméstico }\end{array}$ & 0 & & 0 & & 0 & & 0 & \\
\hline Estilos de Vida & 0 & - - - & 0 & ----- & 0 & $-\cdots$ & 0 & ---. \\
\hline
\end{tabular}

Quadro 3 - Registos dos Enfermeiros com Especialização em Enfermagem de Reabilitação nos diferentes focos adstritos à Ação Realizada pelo Próprio
Em relação à área de atenção autocuidado (Quadro 4), os registos dos enfermeiros com especialização em enfermagem de reabilitação estão essencialmente direcionados para os focos Atividade Física (533 registos - 55,5\%), Autocuidado (271 registos - 17,3\%), Uso do Sanitário (225 registos - 43,1\%), Higiene $(218$ registos - 40,7\%), Vestuário (188 registos - 39,5\%) e Arranjo Pessoal (130 registos - 38,1\%). Relativamente ao foco Comportamento de Procura de Saúde verificaram-se 4 registos e no foco Comportamento de Sono-Repouso 1 registo.

\begin{tabular}{|c|c|c|c|c|c|c|c|c|}
\hline \multirow{2}{*}{$\begin{array}{l}\text { Departa- } \\
\text { mentos } \\
\text { goco re- } \\
\text { gistado } \mathrm{p} / \mathrm{ER}\end{array}$} & \multicolumn{2}{|c|}{ Cirurgia } & \multicolumn{2}{|c|}{ Medicina } & \multicolumn{2}{|c|}{$\begin{array}{l}\text { Medicina } \\
\text { Intensiva }\end{array}$} & \multicolumn{2}{|c|}{ Total } \\
\hline & $\mathrm{n}$ & $\%$ & $\mathrm{n}$ & $\%$ & $n$ & $\%$ & $\mathrm{n}$ & $\%$ \\
\hline Autocuidado & 28 & 11,0 & 106 & 11,2 & 137 & 36,8 & 271 & 17,3 \\
\hline Arranjo pessoal & 5 & 1,9 & 68 & 7,2 & 57 & 15,3 & 130 & 38,1 \\
\hline Beber & 0 & 0,0 & 0 & $\ldots$ & 0 & $-\ldots$ & 0 & 0,0 \\
\hline $\begin{array}{l}\text { Comportamento } \\
\text { de sono-repouso }\end{array}$ & 0 & $-\cdots$ & 1 & 0,10 & 0 & $\mid-\ldots$ & 1 & 50,0 \\
\hline Higiene & 27 & 10,6 & 124 & 13,1 & 67 & 18,0 & 218 & 40,7 \\
\hline Uso do sanitário & 40 & 15,7 & 128 & 13,6 & 57 & 15,3 & 225 & 43,1 \\
\hline Vestuário & 28 & 11,0 & 116 & 12,3 & 44 & 11,8 & 188 & 39,5 \\
\hline Atividade física & 125 & 49,0 & 398 & 42,2 & 10 & 2,7 & 533 & 55,5 \\
\hline $\begin{array}{l}\text { Atividade } \\
\text { recreativa }\end{array}$ & 0 & -.. & 0 & . & 0 & & 0 & \\
\hline Auto agressão & 0 & ---- & 0 & $-\cdots$ & 0 & $-\cdots$ & 0 & $-\cdots$ \\
\hline $\begin{array}{c}\text { Comportamento } \\
\text { de procura de } \\
\text { saúde }\end{array}$ & 2 & 0,78 & 2 & 0,21 & 0 & --- & 4 & 40,0 \\
\hline
\end{tabular}

Quadro 4 - Registos dos Enfermeiros com Especialização em Enfermagem de Reabilitação nos diferentes focos adstritos ao Autocuidado

No âmbito da documentação efetuada em relação ao foco Razão para a Ação, segundo as áreas de especialização, os enfermeiros especialistas em enfermagem de reabilitação são os que apresentam um maior número de registos (89 registos - 65.9\%), nomeadamente no domínio do Auto-conhecimento. O único registo relativo ao Auto-conceito, efetuado por um enfermeiro especialista em enfermagem de reabilitação, refere-se à Auto-estima.

Em relação ao Auto-conhecimento (Quadro 5), segundo as áreas de especialização, os enfermeiros especialistas em enfermagem de reabilitação são os que apresentam um maior número de registos (88 registos ou 65.7\%). Em relação à distribuição dos registos efetuados, segundo as áreas especializações, para os enfermeiros especialistas em enfermagem de reabilitação foram maioritários os focos Memória (26 registos - 57,8\%), Adaptação (23 registos - 71,9\%), Energia (22 registos - 64,7\%) e Cognição (15 registos - 88,5\%). 


\begin{tabular}{|c|c|c|c|c|c|c|c|c|}
\hline \multirow{2}{*}{$\begin{array}{l}\text { Departa- } \\
\text { mentos } \\
\text { Foco re- } \\
\text { gistado } \mathrm{p} / \mathrm{ER}\end{array}$} & \multicolumn{2}{|c|}{ Cirurgia } & \multicolumn{2}{|c|}{ Medicina } & \multicolumn{2}{|c|}{\begin{tabular}{|l|} 
Medicina \\
Intensiva
\end{tabular}} & \multicolumn{2}{|c|}{ Total } \\
\hline & $\mathrm{n}$ & $\%$ & $\mathrm{n}$ & $\%$ & $\mathrm{n}$ & $\%$ & $\mathrm{n}$ & $\%$ \\
\hline Adaptação & 0 & 0,0 & 23 & 85,2 & 0 & $-\cdots$ & 23 & 71,9 \\
\hline Aprendizagem & 0 & -..- & 0 & $-\cdots$ & 0 & $\mid \begin{array}{l}-.- \\
\end{array}$ & 0 & -..- \\
\hline Bem-estar & 0 & 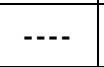 & 0 & $\cdots$ & 0 & $-\cdots$ & 0 & $-\cdots$ \\
\hline Cognição & 0 & 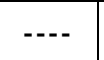 & 1 & 100,0 & 14 & 87,5 & 15 & 88,2 \\
\hline Crença & 0 & $\cdots$ & 0 & $\cdots$ & 0 & $-\cdots$ & 0 & $\ldots$ \\
\hline Emoção & 0 & $-\cdots$ & 2 & 33,3 & 0 & \begin{tabular}{|l|}
--- \\
\end{tabular} & 2 & 33,3 \\
\hline Energia & 2 & 100,0 & 17 & 63,0 & 3 & 60,0 & 22 & 64,7 \\
\hline $\begin{array}{l}\text { Força de } \\
\text { Vontade }\end{array}$ & 0 & $\cdots--$ & 0 & $-\cdots$ & 0 & $-\cdots$ & 0 & $\cdots$ \\
\hline Memória & 13 & 76,5 & 9 & 56,3 & 4 & 33,3 & 26 & 57,8 \\
\hline $\begin{array}{l}\text { Tomada de } \\
\text { Decisão }\end{array}$ & 0 & --.- & 0 & -..- & 0 & -..- & 0 & --.- \\
\hline
\end{tabular}

Quadro 5 - Registos dos Enfermeiros com Especialização em Enfermagem de Reabilitação nos diferentes focos adstritos à Razão para Acção - Auto-conhecimento

Quanto à distribuição dos registos efetuados relativamente ao foco Emoção, segundo as áreas de especialização, a Enfermagem de Saúde Mental e Psiquiátrica é a mais frequente (3 registos - 50,0\%), seguindo-se a Enfermagem de Reabilitação, com 2 registos $(33,3 \%)$ realizados na área Médica.

Em relação à distribuição dos registos relativos à Força de Vontade, os enfermeiros com especialização efetuaram apenas 1 registo, sendo este realizado por um enfermeiro com especialização em Enfermagem de Saúde Mental e Psiquiátrica.

Relativamente aos focos Aprendizagem, Bem-estar, Crença e Tomada de decisão não se verificaram registos de nenhuma das áreas de especialização

\section{DISCUSSÃO}

A utilização de sistemas de informação em suporte eletrónico tem vindo a aumentar de forma significativa, realidade à qual a enfermagem não tem ficado alheia. Nas investigações realizadas por vários autores ${ }^{(6-8)}$, foi possível constatar que o sistema de informação favorece a garantia da continuidade dos cuidados de enfermagem, bem como a visibilidade dos mesmos. Numa investigação realizada no contexto hospitalar(9), constatou-se que os enfermeiros têm consciência da importância do sistema de informação em suporte eletrónico, vislumbrando na sua implementação a possibilidade de dar visibilidade aos cuidados prestados. Neste contexto, importa relembrar que a documentação em enfermagem é uma componente essencial, com significado clínico e legal, sendo que um padrão de documentação pobre contribui para uma pior qualidade de cuidados ${ }^{(10)}$.

Apesar das vantagens de um sistema de informação em suporte eletrónico, com 0 intuito de facilitar a comunicação entre instituições e garantir a possibilidade de comparar dados de enfermagem, em 2007, a Ordem dos Enfermeiros, considerou que um dos princípios básicos ao desenvolvimento do sistema de informação era a utilização da Classificação Internacional para a Prática de Enfermagem $\left(\right.$ CIPE $\left.^{\circledR}\right)$ como referencial de linguagem ${ }^{(11)}$.

Os sistemas de informação têm vindo a ser encarados como um instrumento facilitador da investigação em enfermagem. Relativamente ao referido, tem-se considerado que para além de constituir um recurso estratégico para a gestão, a possibilidade de reutilizar os dados documentados nos sistemas de informação, sob a forma de indicadores sensíveis aos cuidados de enfermagem, abre caminho à consolidação do papel e da importância dos enfermeiros ${ }^{(12)}$ e, particularmente, dos enfermeiros especialistas em enfermagem de reabilitação. Para tal, será necessário, antes de mais, resolver o problema da subdocumentação da assistência de enfermagem ${ }^{(12)}$, e neste caso particular, da enfermagem de reabilitação. A identificação de alguns fatores que possam justificar essa subdocumentação pode constituir um contributo à adoção de estratégias que culminem na otimização do sistema de informação em uso.

No sentido do referido, no âmbito de um estudo realizado no contexto nacional ${ }^{(13)}$, emergiram cinco fatores que condicionam a valorização dos sistemas de informação em suporte eletrónico: as desigualdades entre instituições relativamente à atualização do sistema de informação informatizado; a complexidade do sistema de informação informatizado; a inadequação do sistema de informação informatizado a alguns contextos; o conhecimento sobre o sistema de informação informatizado, bem como o tempo para os registos eletrónicos.

A ideia de que é demasiado o tempo dedicado à documentação, continua a prevalecer entre os enfermeiros. Numa investigação realizada em 2006 constatou-se que as dificuldades inerentes ao manuseamento dos computadores poderiam aumentar o tempo despendido, no entanto, com o treino dessas capacidades, seria possível uma melhor gestão do tempo ${ }^{(6)}$. Para além disso, atendendo aos níveis de literacia tecnológica atuais, julgou-se que as dificuldades inerentes ao manuseamento dos computadores teriam pouca influência ${ }^{(14)}$, o que efetivamente nem sempre acontece.

O problema é que na perspetiva de alguns enfermeiros, independentemente das capacidades para a tecnologia informática, é necessário passar muito tempo ao computador, de modo a corresponder a todas as exigências inerentes à documentação dos cuidados planeados e prestados. Conforme já mencionado em 2006, "o tempo despendido pelos enfermeiros a documentar compete com o tempo disponível para os cuidados diretos aos clientes"(6:18), o que frequentemente culmina numa subdocumentação dos cuidados, neste caso, de enfermagem de reabilitação.

Neste seguimento, a indisponibilidade de tempo também se reflete na falta de atualização dos processos de cuidados no sistema informatizado, existindo frequentemente modificação do status sem atualização dos restantes aspetos incluídos no plano de cuidados. 
É, portanto, notório que o tempo despendido para realizar os registos de enfermagem pode interferir com a qualidade dos mesmos ${ }^{(15)}$.

No âmbito dos fatores que condicionam a documentação dos cuidados de enfermagem, destacam-se a valorização da documentação dos cuidados de enfermagem, bem como o conhecimento sobre a importância da documentação dos cuidados de enfermagem ${ }^{(13)}$.

Tal como já pontuado na literatura, o desafio está em reconhecer a importância dos registos, pois estes espelham o que realmente foi executado, existindo o risco de incorrer na questão, "se não está registado, então não foi executado"(15:34). Uma vez que no estudo que aqui apresentamos foi usada a informação documentada pelos enfermeiros, sobressai a questão: será que os enfermeiros especialistas em enfermagem de reabilitação não documentam os problemas e as necessidades das pessoas no âmbito da morte e dos processos de morrer, ou efetivamente, será uma área menos valorizada no âmbito da sua atuação?

Do mencionado emerge a necessidade de clarificar nos contextos os aspetos inerentes à qualidade informativa da documentação, uma vez que dados incompletos descaracterizam a realidade, a intervenção dos profissionais de saúde e comprometem a adoção de estratégias baseadas na evidência ${ }^{(16,17)}$.

Decorrente da institucionalização da morte, os enfermeiros que exercem a sua atividade profissional em instituições hospitalares, como é o caso dos enfermeiros que participaram neste estudo, deparam-se frequentemente com a necessidade de cuidar de pessoas em processos de morrer e os estudos que têm sido realizados nesses contextos evidenciam que nem sempre se sentem preparados, uma vez que a academia forma o profissional com suporte teórico e prático para preservar a vida, sendo as questões da morte e do morrer pouco ou nada abordadas ${ }^{(18-22)}$. O problema é que, a superficialidade e a banalização das poucas discussões sobre a morte e o processo de morrer não têm permitido a ampliação do entendimento deste fenómeno como um evento integrante do ciclo de vida $^{(19)}$

$\mathrm{Na}$ sequência do mencionado e atendendo aos resultados obtidos neste estudo, torna-se emergente que os enfermeiros se descentrem de uma prática predominantemente focada na satisfação das necessidades humanas básicas, em prol de um cuidado centrado nas experiências vivenciadas pelas pessoas diante da iminência e inevitabilidade da finitude da vida, com potencial para culminar numa morte digna e tranquila $^{(23)}$.

Quando analisados os registos, segundo as áreas de especialização, os enfermeiros especialistas em enfermagem de reabilitação são os que apresentam um maior número de registos no âmbito da "Pessoa" e da "Ação realizada pelo Próprio", o que evidencia, mais uma vez, o valor atribuído por estes enfermeiros especialistas ao projeto individual de cada pessoa.

A documentação efetuada no âmbito da função, denota que apesar do valor atribuído ao domínio da pessoa, os enfermeiros especialistas em enfermagem de reabilitação dão especial enfoque a três áreas de atenção: tegumento, atividade motora e respiração.

Atendendo às características do exercício profissional dos enfermeiros especialistas em enfermagem de reabilitação, o enfoque no tegumento está perfeitamente integrado na prevenção de complicações. Por outro lado, a atuação centrada na atividade motora e na respiração, evidenciam o contributo do enfermeiro especialista em enfermagem de reabilitação na maximização do potencial da pessoa, sendo que para tal concebe, implementa e monitoriza planos de enfermagem de reabilitação diferenciados, baseados nos problemas reais e potenciais das pessoas em três componentes essenciais: motora, neurológica e cardiorrespiratória.

Uma vez que os cuidados prestados no âmbito da morte e dos processos de morrer, necessitam de estar ancorados em referenciais teóricos de enfermagem ${ }^{(23)}$, dos resultados relativos ao foco "Razão para a ação" emerge a necessidade de se repensarem as práticas. De facto, a ausência de registos no âmbito dos focos aprendizagem, bem-estar, crença e força de vontade, cruciais no âmbito de alguns dos referenciais teóricos de enfermagem, releva a necessidade da conceção e respetiva documentação dos cuidados contemplar estas áreas, essenciais durante a vivência das transições. Para além disso, a inclusão destas áreas de atenção garantirá que os enfermeiros sejam capazes de reconhecer, não apenas os aspetos biológicos, muito centrados na função, mas também as implicações psicossocioespitituais da vivência da morte e dos processos de morrer nos doentes, nas famílias e nos familiares cuidadores ${ }^{(23)}$.

\section{CONCLUSÃO}

As instituições hospitalares têm sofrido alterações significativas, orientadas quer para a satisfação das necessidades dos clientes, quer para a qualidade do serviço prestado. Em virtude de tais exigências, a correta documentação dos problemas e necessidades dos clientes é imprescindível ao sucesso de qualquer organização.

No entanto, apesar da relevância atualmente atribuída à documentação dos cuidados de enfermagem de reabilitação, existem algumas fragilidades, principalmente em relação à atualização do processo de cuidados. De facto, apesar de se identificarem os problemas e as necessidades dos clientes no âmbito da morte e dos processos de morrer, nem sempre ocorre o seu registo, o que suscita a dúvida quanto à sua concretização.

Embora seja conhecido que nem sempre a atuação dos enfermeiros é congruente com os referenciais teóricos da disciplina e com os instrumentos reguladores do exercício profissional, vários estudos têm comprovado que o executado é significativamente superior ao registado. Perante o referido, para além de ser crucial a documentação efetuada pelos enfermeiros, seria interessante a realização de estudos qualitativos com intuito de identificar os fatores que facilitam ou comprometem uma atuação congruente com os instrumentos reguladores da profissão e com os 
referenciais teóricos da disciplina e/ou que por outro lado, comprometam a documentação do planeado e executado. Seria da mesma forma interessante, embora não no âmbito do projecto "Viver a morte: desafio da profissão de enfermagem", comparar estes resultados com os registos efetuados pelos enfermeiros especialistas de enfermagem de reabilitação nos restantes clientes.

\section{REFERÊNCIAS BIBLIOGRÁFICAS}

1. Sousa DM, Soares EO, Costa KM, Pacifico AL, Parente AC. A vivência da enfermeira no processo de morrer dos pacientes oncológicos. Texto Contexto Enferm [Internet]. 2009 [cited 2019 20 jan];18(1): 41-47. Disponível em: http://www.scielo.br/pdf/tce/v18n1/v18n1a05.pdf

2. Brito FM, Costa IC, Costa SF, Andrade CG, Santos KF, Francisco DP. Communication in death imminence: perceptions and strategy adopted for humanizing care in nursing. Esc. Anna Nery [Internet]. 2014 [cited $2019 \mathrm{fev} 6$ ];18 ${ }^{(2)}:$ 317-322. Disponível em: http://www.scielo.br/pdf/ean/v18n2/en_1414-8145-ean-18-020317.pdf

3. Portugal. Ministério da Saúde. Regulamento n. ${ }^{\circ} 140 / 2019$, de 6 de fevereiro de 2019. Regulamento das Competências Comuns do Enfermeiro Especialista. Diário da República. 2019 fev; 2. série. $p$ 4744-4750. Disponível em: https://www.ordemenfermeiros.pt/media/10778/0474404750.pdf

4. Portugal. Ministério da Saúde. Regulamento n. ${ }^{\circ} 392 / 2019$, de 03 de maio de 2019. Regulamento das Competências Específicas do Enfermeiro Especialista em Enfermagem de Reabilitação. Diário da República. 2019 maio; 2. ${ }^{a}$ série. p 13565-13568. Disponível em: /search/122216893/details/normal?l=1 https://dre.pt/web/guest/pesquisa/-

5. Martins MM, Ribeiro OM, Silva JV. Orientações concetuais dos enfermeiros especialistas em enfermagem de reabilitação em hospitais portugueses. Rev Port Enferm de Reabil [Internet]. 2018 [cited 201920 fev]; $2^{(1)}$ : 42-48. Disponível em: https://www.aper.pt/Ficheiros/Revista/RPERV1N2.pdf

6. Silva AA. Sistemas de Informação em Enfermagem - Uma Teoria Explicativa da Mudança. Coimbra: FORMASAU - Formação e Saúde, Lda; 2006.

7. Sousa PA. Sistema de Partilha de Informação de Enfermagem entre Contextos de Cuidados de Saúde: um Modelo Explicativo. Coimbra: FORMASAU - Formação e Saúde, Lda; 2006.

8. Mota LA, Pereira FM, Sousa PA. Nursing Information Systems: exploration of information shared with physicians. Rev Enferm Ref [Internet]. 2014 [cited 2019 Jan 29];(1):85-91. Available from: http://www.scielo.mec.pt/pdf/ref/vserlVn1/serlVn1a10.pdf

9. Pinto LF. Sistemas de informação e profissionais de enfermagem [Dissertação de Mestrado não publicada]. Vila Real: Universidade de Trás-os-Montes e Alto Douro; 2009.

10. Prideaux A. Issues in nursing documentation and record-keeping practice. $\mathrm{Br} J$ Nurs. [Internet]. 2011 [cited 2019 Jan $21] ; 20^{(22)}: 1450-1454 . \quad$ Available $\quad$ from: https://www.ncbi.nlm.nih.gov/pubmed/22241496

11. Ordem dos Enfermeiros. Sistema de Informação em Enfermagem - Princípios básicos da arquitectura e principais requisitos técnico-funcionais; $2007 . \quad$ Disponível em: http://www.ordemenfermeiros.pt/documentosoficiais/Documents/ SIE-PrincipiosBasicosArq_RequisitosTecFunc-Abril2007.pdf

12. Leite FG. Sistemas de Informação: Contributos para a definição de um painel de indicadores do exercício profissional dos enfermeiros num Departamento de Medicina. [Dissertação de Mestrado]. Porto: Escola Superior de Enfermagem do Porto; 2016.

13. Ribeiro $\mathrm{OM}$. Concecões e práticas dos enfermeiros: olhares sobre um percurso em hospitais portugueses. Loures: Lusodidacta; 2018.

14. Campos AM. Satisfação dos Utilizadores de Sistemas de Informação e Documentação de Enfermagem em Suporte Eletrónico: Um Estudo no Centro Hospitalar de Coimbra, E.P.E. [Dissertação de Mestrado]. Coimbra: Universidade de Coimbra,
Faculdade de Economia; 2012.

15. Tareco ES. Sistemas Informáticos para a Qualidade dos Cuidados de Enfermagem, Revisão Sistemática [Dissertação de Mestrado]. Faro: Universidade do Algarve, Faculdade de Economia; 2015.

16. Ministério da Saúde. A Organização Interna e a Governação dos Hospitais. Lisboa: Ministério da Saúde; 2010.

17. Machado NJ. Gestão da Qualidade dos Cuidados de Enfermagem - Um Modelo de Melhoria Contínua Baseado na Reflexão-Ação [Tese de Doutoramento]. Porto: Instituto de Ciências Biomédicas Abel Salazar, Universidade Católica Portuguesa; 2013.

18. Alves AC. Ensino de Teorias de Enfermagem na Formação Profissional do Enfermeiro [Dissertação de Mestrado]. Itajubá: Escola de Enfermagem Wenceslau Braz; 2012.

19. Dias MV, Backes DS, Barlem EL, Backes MT, Lunardi VL, Souza $M H$. Nursing undergraduate education in relation to the deathdying process: perceptions in light of the complex thinking. Rev Gaúcha Enferm [Internet]. 2014 [cited 2019 Jan 11];35(4):79-85. Available from: http://dx.doi.org/10.1590/19831447.2014.04.45177.

20. Andersson E, Salickiene Z, Rosengren K. To be involved - A qualitative study of nurses' experiences of caring for dying patients. Nurse Educa Today [Internet]. 2016 [cited 2019 Jan 14];38:144-149. Available from: https://www.ncbi.nlm.nih.gov/pubmed/26689734

21. Salimena AM, Ferreira GC, Melo MC. Feelings of surgical nursing staff in face of death. Arq Ciênc Saúde. 2015 [cited 2019 Jan 9];22(1):75-78. Available from: https://doi.org/10.17696/23183691.22.1.2015.33

22. Mota MS, Gomes GC, Coelho MF, Lunardi Filho WD, Sousa LD. Reactions and feelings of nursing professionals facing death of patients under their care. Rev Gaúcha Enferm [Internet]. 2011 [cited 2019 Jan 3];32 $2^{(1)}: 129-135$. Available from: http://dx.doi.org/10.1590/S1983-14472011000100017.

23. Cardoso MF, Ribeiro OM, Martins MM. Death and dying: contributions to a practice based on nursing theoretical frameworks. Rev Gaúcha Enferm [Internet]. 2019 [cited 2019 fev 27]; 40:e201 